\title{
Etude de composés volatils neutres présents dans le Vacherin
}

\author{
par \\ J. P. DUMONT, Sylviane ROGER, Paule CERF \\ et J. ADDA \\ Laboratoire d'Etude des Arômes. C.N.R.Z. - 78350 Jouy-en-Josas
}

\section{I. - INTRODUCTION}

Dans un travail publié récemment [1] nous avons mis en évidence un certain nombre de composés neutres participant à l'arôme de différents types de fromages à pâte molle et à croûte lavée. Désirant conserver à cette étude un caractère homogène, nous avions alors écarté le "Vacherin » (également appelé "Mont d'Or » ou « fromage de boîte ") qui se distingue sensiblement, tant du point de vue organoleptique que du point de vue affinage, de fromages comme le Livarot ou le Pont-l'Evêque et qui nous a semblé justifier une étude particulière. Une publication très récente [2] apporte quelques données concernant le "Vacherin " mais ne fait état que de l'identification de méthyl-cétones et d'alcools secondaires ; ceci ne permet pas, de l'aveu même des auteurs, d'expliquer la flaveur typique de ce fromage.

L'existence d'une croûte bien individualisée et l'influence présumée du contact prolongé entre cette croûte et la lanière d'écorce de résineux qui enserre le fromage nous ont amené à étudier séparément le cœur et la croûte espérant ainsi mettre en évidence la migration de produits de la croûte vers le cœur.

\section{II. - MATERIEL ET METHODES}

\section{a) Les fromages}

Trois des fromages étudiés (échantillons 1 à 3 ) provenaient du Jura français et étaient présentés de façon traditionnelle, cerclés d'une lanière d'écorce de résineux et placés dans une boîte en bois blanc avec laquelle ils étaient directement en contact. L'échantillon 1 âgé de 1 mois au moment de l'examen avait été fabriqué par l'Ecole 
Nationale d'Industrie Laitière de Poligny à partir de lait thermisé à $68^{\circ} \mathrm{C}$ pendant quelques secondes ; l'échantillon 2 âgé de 1 mois avait été fabriqué dans une fruitière de montagne à partir de lait cru ; l'échantillon 3 provenant du département du Doubs avait été fabriqué à partir de lait chauffé plusieurs minutes à $58-62^{\circ} \mathrm{C}$; l'échantillon 4 , d'origine inconnue, avait été acheté sur un marché de la région parisienne. Ce dernier échantillon présentait à la dégustation un défaut marqué de flaveur (flaveur fécale), qui le rendait pratiquement inconsommable. Les échantillons 2' et 3' correspondent respectivement à la croûte des fromages 2 et 3 .

\section{b) Extraction}

Les fromages écroûtés sont congelés puis râpés. Un échantillon de $125 \mathrm{~g}$ environ est soumis à une distillation sous vide poussé suivant une technique précédemment décrite [1]. La croûte est traitée indépendamment mais de façon similaire.

\section{c) Analyse chromatographique}

Les analyses ont été effectuées sur un appareil Girdel 3000 , à détecteur à ionisation de flamme équipé d'une colonne en acier inoxydable de $3 \mathrm{~m}$ de long et de $1 / 8$ de pouce de diamètre externe remplie de chromosorb G (60-80 mesh) AW-DMCS, imprégné de 5 p. 100 de Carbowax 20 M-TPA (Applied Sciences) purifié par nos soins. La température du four augmente de façon linéaire à raison de $2^{\circ} \mathrm{C}$ par mn de $50^{\circ} \mathrm{C}$ à $165^{\circ} \mathrm{C}$ après une période isotherme de $10 \mathrm{mn}$ à $50^{\circ} \mathrm{C}$. Cette première analyse permet de juger de la richesse de l'extrait en composés volatils.

Une seconde analyse est effectuée sur un appareil Girdel 75 CD PT modifié de façon à obtenir le tracé de la détection par ionisation de flamme tout en évaluant simultanément l'intensité et la qualité olfactive des différents composés séparés en sortie de colonne (cette modification permet éventuellement le piégeage de différentes fractions).

\section{d) Spectrométrie de masse}

Les extraits sont également analysés sur un appareil Girdel 3000 couplé à un spectromètre de masse à faible résolution (AEI MS 20 organique).

La séparation chromatographique est réalisée sur une colonne capillaire d'une longueur de $100 \mathrm{~m}$ et d'un diamètre intérieur de $1 \mathrm{~mm}$, préparée par nos soins en utilisant comme phase stationnaire un mélange de Silicone SF 96 et d'Igepal CO-880 dans les proportions 95:5. Le gaz vecteur est l'hélium qui circule à un débit de $8 \mathrm{ml} / \mathrm{mn}$. 
La température est programmée de $30^{\circ} \mathrm{C}$ à $140^{\circ} \mathrm{C}$ à raison de $1^{\circ} \mathrm{C} / \mathrm{mn}$ après une période isotherme de $10 \mathrm{mn}$ à $30^{\circ} \mathrm{C}$.

Les spectres sont enregistrés à $70 \mathrm{eV}$ et identifiés en ayant recours aux données publiées par Cornu et Massot [3].

\section{III. - RESULTATS}

D'une façon générale le «Vacherin » fournit un extrait pauvre en produits volatils. Les composés identifiés dans les divers échantillons sont présentés dans les tableaux 1 à 8 , où nous avons essayé de donner une idée de l'importance quantitative de chacun des composés en utilisant une échelle de cotation de 1 à $4(+$ à ++++$)$.

\section{IV. - DISCUSSION}

En ce qui concerne les alcools aliphatiques (tab. 1) on peut voir que les composés constamment présents dans le cœur sont le pro-

TABLEAU 1. - Alcools aliphatiques

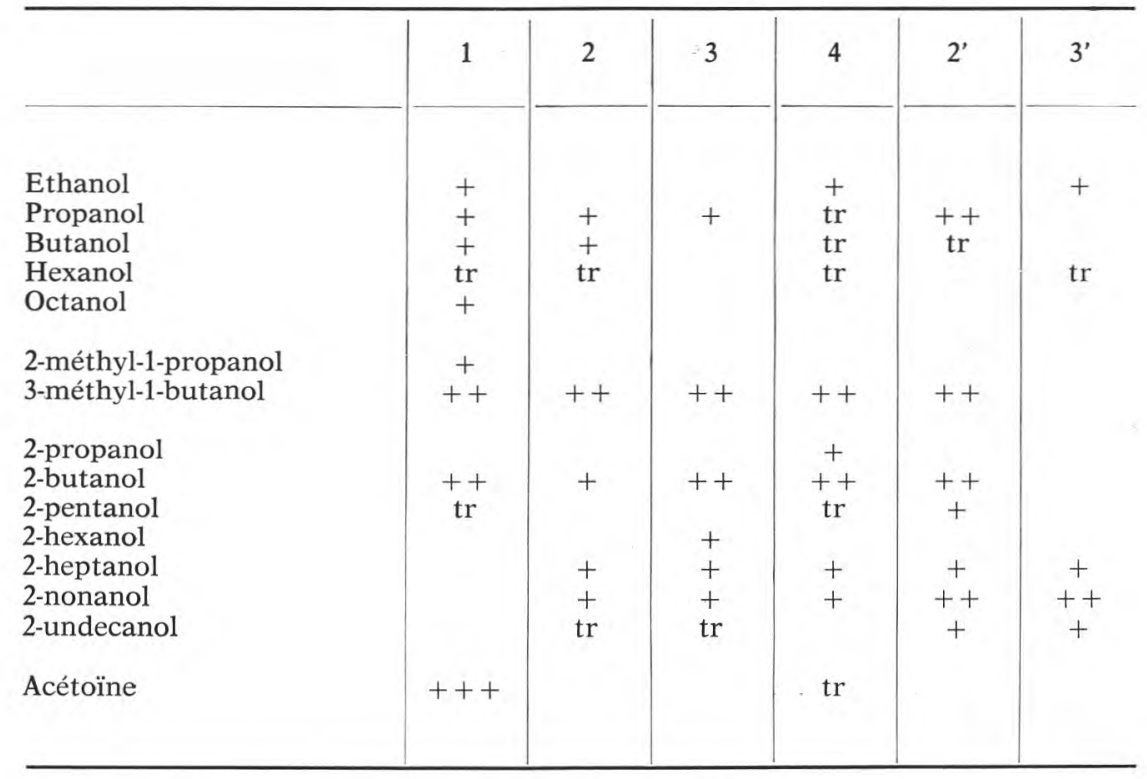

panol, le 3-méthyl-1-butanol et le 2 butanol. Le 2-heptanol et le 2-nonanol sont absents de l'échantillon 1, qui d'une façon générale est moins riche en composés volatils mais qui en revanche est le 
TABLEAU 2. - Cétones et aldéhydes aliphatiques

2-butanone

2-pentanone

3-pentanone

2-hexanone

2-heptanone

3-octanone

2-nonanone

2-undecanone

2-tridecanone

2-nonenone

Hexanol

Heptanol

2-méthyl butanol

3-méthyl butanol

Méthyl-2-butène-2-al

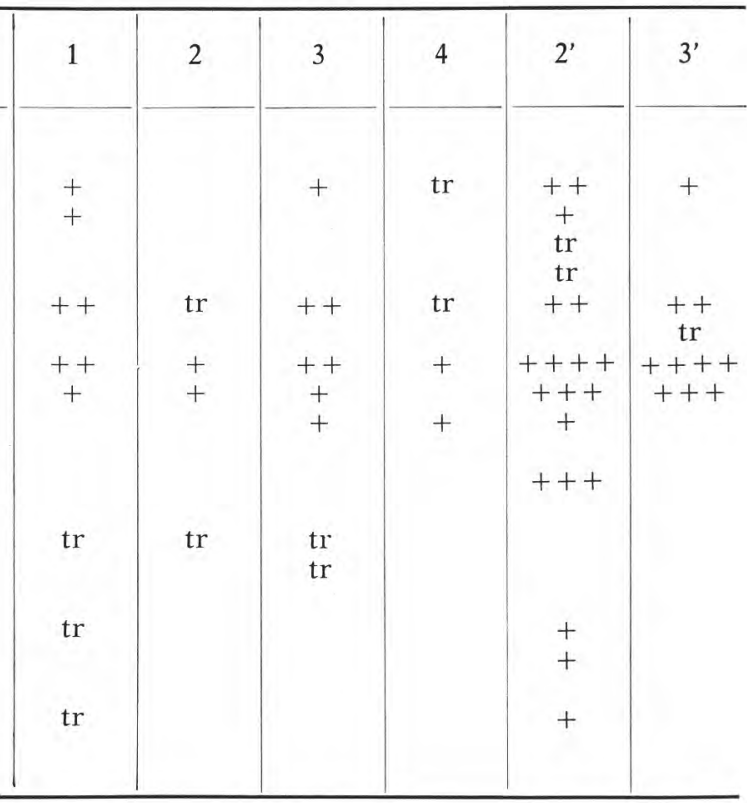

TABLEAU 3. - Esters aliphatiques

Tétradécanoate de méthyle Hexadécanoate de méthyle

Acétate d'éthyle

Butanoate d'éthyle

Hexanoate d'éthyle

Octanoate d'éthyle

Décanoate d'éthyle

Dodécanoate d'éthyle

Tétradécanoate d'éthyle

Acétate d'isoamyle

Acétate de butyle

The

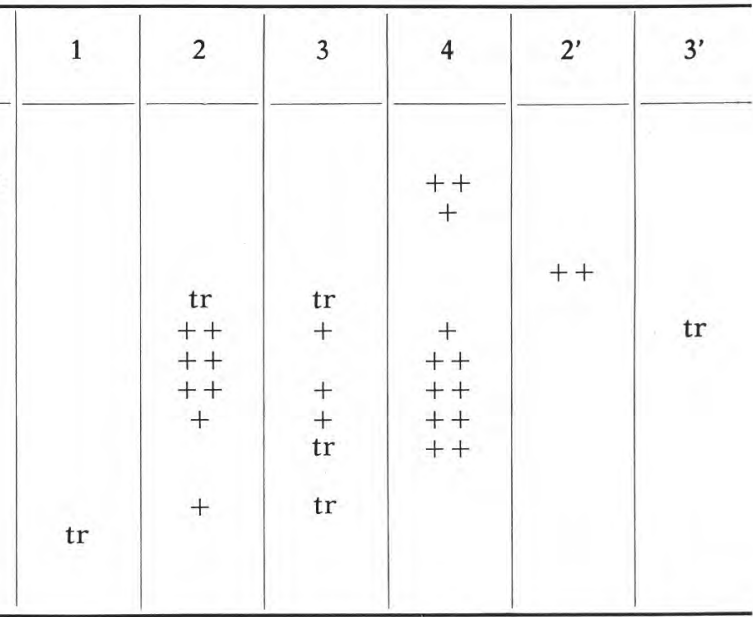

seul à contenir de l'acétoïne. On peut noter que le 3-méthyl-1-butanol était un composé toujours présent en quantités importantes dans les fromages à croûte lavée étudiés antérieurement et que sa présence concorde bien avec l'existence de quantités importantes d'acide isovalérique parmi les acides gras volatils du "Vacherin ». (Les autres 
acides mis en évidence sont l'acétique, le propionique en quantité importante, l'isobutyrique, le butyrique, l'isocaproïque et le caproïque).

Le tableau 2 résume les résultats obtenus pour les cétones et les aldehydes aliphatiques; ils sont qualitativement en accord avec ceux obtenus par Groux et Moinas [2] mais en diffèrent quantitativement, surtout au niveau des cétones à longue chaîne. Ces différences peuvent s'expliquer par l'utilisation de méthodes d'extraction très différentes, la méthode que nous utilisons permettant sans doute de mieux extraire les composés peu volatils.

Par ailleurs, il apparaît que les échantillons 2' et 3' (croûtes) sont très riches en méthylcétones à longue chaîne.

Le tableau 3 présente les esters aliphatiques mis en évidence. Il faut remarquer, pour les échantillons 2 et 3 , une nette opposition entre le cœur assez riche en esters et la croûte qui n'en contient pas.

Les composés aromatiques (tab. 4) représentent une part importante des composés volatils. Le 2-phényl éthanol et le phénol présents dans les échantillons 1 à 4 étaient également toujours présents dans

TABLEAU 4. - Composés aromatiques

\begin{tabular}{|c|c|c|c|c|c|c|}
\hline & 1 & 2 & 3 & 4 & $2^{\prime}$ & $3^{\prime}$ \\
\hline $\begin{array}{l}\text { Benzaldéhyde } \\
\text { Acétophénone }\end{array}$ & $\begin{array}{l}\operatorname{tr} \\
++\end{array}$ & & & ++ & +++ & \\
\hline $\begin{array}{l}\text { 2-phényl éthanol } \\
\text { 2-phényl éthane-2-o1 }\end{array}$ & + & + & + & $\begin{array}{c}++ \\
+\end{array}$ & & \\
\hline $\begin{array}{l}\beta \text {-phényl éthyl acétate } \\
\beta \text {-phényl éthyl propanoate }\end{array}$ & & $\begin{array}{c}++ \\
++\end{array}$ & $\operatorname{tr}$ & & & \\
\hline $\begin{array}{l}\text { Phénol } \\
\text { Crésol } \\
\text { Anisol }\end{array}$ & +++ & +++ & + & $\begin{array}{l}++++ \\
++++\end{array}$ & $\begin{array}{c}++ \\
++\end{array}$ & \\
\hline $\begin{array}{l}\text { Benzène } \\
\text { Toluène } \\
\text { Ethyl benzène } \\
\text { Diméthyl benzène } \\
\text { Ethyl méthyl benzène } \\
\text { Triméthyl benzène } \\
\text { Diméthyl éthyl benzène } \\
\text { Tétraméthyl benzène } \\
\text { Styrène }\end{array}$ & $\begin{array}{l}\mathrm{tr} \\
\mathrm{tr} \\
+ \\
+ \\
\mathrm{tr} \\
+\end{array}$ & $\begin{array}{l}\operatorname{tr} \\
\operatorname{tr} \\
\operatorname{tr} \\
\operatorname{tr} \\
\operatorname{tr} \\
+ \\
\operatorname{tr}\end{array}$ & $\begin{array}{l}+ \\
\mathrm{tr} \\
\mathrm{tr} \\
\mathrm{tr} \\
\mathrm{tr}\end{array}$ & $\begin{array}{l}\operatorname{tr} \\
\operatorname{tr}\end{array}$ & $\begin{array}{c}++ \\
\operatorname{tr} \\
+ \\
\operatorname{tr} \\
+ \\
++\end{array}$ & $\begin{array}{l}\operatorname{tr} \\
\text { tr } \\
\text { tr } \\
+ \\
\text { tr } \\
+ \\
+ \\
+ \\
+\end{array}$ \\
\hline $\begin{array}{l}\text { Naphtalène } \\
\text { Méthyl naphtalène }\end{array}$ & & & & & + & + \\
\hline
\end{tabular}


les autres fromages à croûtes lavées étudiés précédemment [1]. On remarquera que le crésol est présent seulement dans l'échantillon 4 qui, comme nous l'avons dit, présentait un défaut d'arôme caractérisé. L'examen olfactif en sortie de colonne a permis d'établir une relation entre la présence de ce composé et l'existence du défaut. Ce résultat est à rapprocher des résultats de Badings et al. [4] qui signalent le rôle que le crésol peut jouer dans l'apparition d'une flaveur putride (également décrite comme phénolique) dans certains lots de fromage de Gouda. Ces auteurs retiennent l'hypothèse qui attribue la présence du crésol à une contamination bactérienne de la présure. Nous ne pouvons pas affirmer que l'origine du crésol présent dans notre échantillon soit la même mais une telle explication n'est pas à écarter.

L'existence de nombreux hydrocarbures benzéniques substitués, présents à l'état de traces, est à remarquer. Le fait que ces composés n'apparaissent pas systématiquement dans toutes les analyses effectuées au laboratoire semblerait exclure l'hypothèse d'une contamination, sans que nous puissions pour autant expliquer l'origine de ces composés.

Les composés soufrés identifiés (tab. 5) sont les mêmes que ceux identifiés précédemment dans d'autres fromages à croûte lavée. On remarquera que le disulfure de méthyle et le 2,3-4 trithiapentane sont présents dans tous les échantillons.

TABLEAU 5. - Composés soufrés

\begin{tabular}{|c|c|c|c|c|c|c|}
\hline & 1 & 2 & 3 & 4 & $2^{\prime}$ & $3^{\prime}$ \\
\hline $\begin{array}{l}\text { Diméthyl disulfure } \\
\text { Ethyl méthyl disulfure } \\
\text { 2,3,4-trithiapentane } \\
\text { Méthylthiol acétate } \\
\text { Méthylthiol propanoate }\end{array}$ & $\begin{array}{c}++ \\
++ \\
++ \\
+ \\
+\end{array}$ & $\begin{array}{l}+ \\
+ \\
\text { tr }\end{array}$ & $\begin{array}{c}++ \\
+\end{array}$ & $\begin{array}{l}++ \\
\operatorname{tr} \\
\operatorname{tr}\end{array}$ & $\begin{array}{c}+++ \\
+ \\
+\end{array}$ & $\begin{array}{l}+++ \\
++\end{array}$ \\
\hline
\end{tabular}

L'originalité du «Vacherin » réside dans la présence d'une série de composés que nous n'avions pas mis en évidence dans les fromages à pâte molle à croûte lavée. Ces substances, de caractère terpénique (tab. 6) sont présentes en grandes quantités dans la croûte ; ceci laisse à penser qu'ils proviennent de la lanière de résineux qui enserre le fromage. Cependant, il semble que la migration vers le cœur soit très inégale selon les composés. Il faut remarquer que les corps qui présentent une fonction alcool semblent migrer plus facilement que ceux qui possèdent le caractère hydrocarbure. 
TABLEAU 6. - Terpènes

\begin{tabular}{|c|c|c|c|c|c|c|}
\hline & 1 & 2 & 3 & 4 & $2^{\prime}$ & $3^{\prime}$ \\
\hline $\begin{array}{l}\text { Camphor } \\
\text { Fenchone }\end{array}$ & $\begin{array}{c}+ \\
++\end{array}$ & & $\begin{array}{l}+ \\
+\end{array}$ & $\begin{array}{l}\operatorname{tr} \\
\operatorname{tr}\end{array}$ & $\begin{array}{c}+ \\
++\end{array}$ & $\begin{array}{c}+ \\
++\end{array}$ \\
\hline $\begin{array}{l}\text { Limonène } \\
\text { Terpinène } \\
\text { Myrcène } \\
\beta \text {-pinène } \\
\text { m/e 121-93-136-68 } \\
\text { Allocimène }\end{array}$ & $\begin{array}{l}+ \\
\operatorname{tr} \\
\operatorname{tr}\end{array}$ & & $\begin{array}{l}+ \\
+\end{array}$ & & $\begin{array}{c}+++ \\
++ \\
++ \\
+++ \\
++\end{array}$ & $\begin{array}{c}+++ \\
+ \\
+++ \\
++++ \\
++\end{array}$ \\
\hline $\begin{array}{l}\text { Isobornéol } \\
\text { Linalool } \\
\alpha \text {-terpinéol } \\
\text { Thymol }\end{array}$ & $\begin{array}{c}++ \\
+++ \\
++++\end{array}$ & $\begin{array}{c}+++ \\
+++ \\
++++ \\
\operatorname{tr}\end{array}$ & $\begin{array}{c}+++ \\
+++ \\
++++ \\
+\end{array}$ & $+++t$ & $\begin{array}{c}+++ \\
+++ \\
++++\end{array}$ & ++++ \\
\hline
\end{tabular}

TABLEAU 7. - Composés chlorés

Chloroforme

Tétrachlorure de carbone Trichloroéthylène Tétrachloroéthylène

Dichlorobenzène Trichlorobenzène

\begin{tabular}{|c|c|c|c|c|c|}
\hline 1 & 2 & 3 & 4 & $2^{\prime}$ & $3^{\prime}$ \\
\hline $\begin{array}{c}+++ \\
\operatorname{tr}\end{array}$ & ++++ & +++ & $\mathrm{tr}$ & & $\begin{array}{c}++ \\
+\end{array}$ \\
\hline $\begin{array}{l}+ \\
\operatorname{tr}\end{array}$ & $\mathrm{tr}$ & $\begin{array}{l}+ \\
\text { tr }\end{array}$ & & + & $\mathrm{tr}$ \\
\hline
\end{tabular}

Compte tenu des seuils de perception relativement élevés des terpènes [6], il est difficile de définir quelle est leur contribution exacte à l'arôme particulier du Vacherin. Néanmoins, comme ils constituent les seuls éléments qui différencient nettement le "Mont d'Or " des autres fromages à pâte molle et à croûte lavée, on peut raisonnablement penser qu'ils présentent une réelle importance.

Enfin, on peut rappeler que Langler [5] a mis en évidence des terpènes ( $\alpha$ pinène et fenchène) dans le "Swiss cheese ". Dans ce cas particulier il est probable que ces composés ont eu le lait pour origine, bien que le contact avec le moule en bois qui, dans le procédé de fabrication traditionnel, enserre le fromage pendant le pressage ne soit pas à écarter complètement. 
On a regroupé dans le tableau 8 des composés à structures diverses parmi lesquelles l'indole est le plus important quantitativement. L'existence d'une pyrazine dans l'échantillon 2' est un fait intéressant à noter même s'il s'agit d'une trace. En effet, ces composés ont un seuil de perception extrêmement bas. L'existence de pyrazines a été mise en évidence par de nombreux auteurs dans des produits laitiers chauffés, mais le fait que l'échantillon 2 provienne d'un fromage de lait cru est à rapprocher d'autres résultats [7] où ce type de composés a été mis en évidence dans la zone corticale d'un échantillon de fromage de Comté.

TABLEAU 8. - Autres composés

\begin{tabular}{|c|c|c|c|c|c|c|}
\hline & 1 & 2 & 3 & 4 & $2^{\prime}$ & $3^{\prime}$ \\
\hline $\begin{array}{l}\text { 8-décalactone } \\
\delta \text {-dodécalactone }\end{array}$ & +++ & +++ & $\begin{array}{l}+++ \\
+++\end{array}$ & & + & \\
\hline $\begin{array}{l}\text { Indole } \\
\text { Méthyl indole }\end{array}$ & ++++ & $\underset{\operatorname{tr}}{+++}$ & $\underset{\text { tr }}{++}$ & ++++ & ++++ & \\
\hline $\begin{array}{l}\text { Triméthyl pyridine } \\
\text { Diméthyl pyrazine }\end{array}$ & + & $\mathrm{tr}$ & $\operatorname{tr}$ & & $\mathrm{tr}$ & \\
\hline $\begin{array}{l}\text { Octane } \\
\text { Nonane } \\
\text { Décane } \\
\text { Undécane } \\
\text { Tridécane }\end{array}$ & $\begin{array}{l}+ \\
+\end{array}$ & $\begin{array}{l}+ \\
+\end{array}$ & + & & $\begin{array}{l}\text { tr } \\
++ \\
++ \\
++\end{array}$ & $\begin{array}{l}\text { tr } \\
+ \\
+ \\
\text { tr }\end{array}$ \\
\hline $\begin{array}{l}\text { 2-méthyl pentane } \\
\text { 3-méthyl pentane }\end{array}$ & & & & & $\begin{array}{l}+ \\
+\end{array}$ & \\
\hline
\end{tabular}

La comparaison du fromage 2 (fabriqué à partir de lait cru) avec les fromages 1 et 3 (fabriqués à partir de lait ayant subi un traitement thermique) ne met pas en évidence de différences qualitatives sensibles : on peut juste remarquer que l'échantillon 1 est de façon générale moins riche en composés volatils. Il est possible d'en conclure que les traitements thermiques modérés appliqués au lait lors de la fabrication des fromages 1 et 3 sont satisfaisants au plan de l'arôme puisqu'ils n'ont pas entraîné de modifications trop importantes dans le profil aromatique général du fromage. Cependant, on peut penser que pour d'autres fabrications fromagères ne bénéficiant pas de laits d'une aussi bonne qualité bactériologique, l'utilisation de barèmes thermiques plus sévères conduit à des différences notables entre fromages produits à partir de lait cru et fromages fabriqués à partir de lait pasteurisé. 


\section{Rés u m é}

On a comparé les produits neutres volatils, obtenus par distillation sous vide poussé et identifiés par couplage chromatographie en phase gazeuse - spectrométrie de masse, à partir de fromages Vacherin - Mont-d'Or fabriqués soit avec du lait cru, soit avec du lait ayant subi un léger traitement thermique. En plus des produits considérés comme importants dans l'arôme des fromages à pâte molle et à croûte lavée (3-méthyl-1-butanol, 2-phényl éthanol, disulfure de méthyle et phénol), on a trouvé une quantité importante de composés terpéniques qui semblent provenir de la lanière d'écorce de résineux qui enserre le fromage durant l'affinage.

\section{S u m m a ry}

Neutral volatiles compounds obtained from three samples of Vacherin - Mont-d'Or, made from raw or low heated milk have been studied by GC-MS-techniques. The core and the rind are studied separately. It has been found that as on others types of surface ripened cheeses 3-methyl-1-butanol, 2-phenyl ethanol, dimethyl disulfide and phenol are important. Besides quite a large quantity of terpenes have been identified. The ring of spruce wood which is put around the cheese during its ripening could be responsible for this.

Reçu pour publication le 23 mars 1974.

\section{Références}

[1] Dumont (J. P.), Roger (Sylviane), AdDA (J.) (1974). - Etude des composés volatils neutres présents dans les fromages à pâte molle et à croûte lavée. Le Lait, LIV, 531-532, 31 .

[2] Groux (M.), Moinas (M.). (1974). - La flaveur des fromages. 2. Etude comparative de la fraction volatile neutre de divers fromages. Le Lait, LIV, 531$532,44$.

[3] Cornu (A.), Massot (R.) (1966). - Index de spectres de masse. P.U.F.

[4] Badings (H. T.), Stadhouders (J.), Van Duin (H.) (1968). - Phenolic flavor in cheese. J. Dairy Sci., 51, 1, 31 (1968).

[5] Langler (J. E.), LibBey (L. M.), Day (E. A.) (1967). - Identification and evaluation of selected compounds in Swiss cheese flavor. J. Agr. Food Chem., $15,3,386$.

[6] Ruthe (M.), Wolm (G.), Tunger (L.), Siebert (H. J.) (1972). - SchwellenKonzentrationen von Aromastoffen und ihre Nutzung zur Auswertung von Aromaanalysen. Nahrung, 16 (5), 483.

[7] Dumont (J. P.), AdDA (J.). - Résultats non publiés. 